Rev. Psicol. (Arequipa. Univ. Catól. San Pablo) / Año 2019 / Vol 9 / № 3 / pp. 75-9o

ISSN 2306-0565 versión impresa / ISSN 2311-7397 versión on line

(c) $(9)$

Esta obra está bajo una Licencia Creative Commons

Atribución 4.o Internacional (BY-NC-ND)

\title{
CLIMA AFECTIVO PARA EL TRABAJO COOPERATIVO DE DOCENTES DE LIMA
}

\section{AFFECTIVE CLIMATE FOR THE COOPERATIVE WORK OF TEACHERS IN LIMA}

Doris Elida Fuster-Guillén', Liliana Hilda Aldazábal Melgar², Sara Ynes Tello Cabello3, Patricia Edith Guillén Aparicio3, Ronald M. Hernández ${ }^{4}$

1. Universidad Nacional Mayor de San Marcos, Lima, Perú

2. Universidad César Vallejo, Lima, Perú

3. Universidad San Martín de Porres, Lima, Perú

4. Universidad San Ignacio de Loyola, Lima, Perú

\begin{abstract}
Resumen
La investigación clima afectivo para el trabajo cooperativo de los docentes nace tras la inquietud que aqueja a las instituciones educativas respecto a las responsabilidades de los agentes educativos, cuyo propósito fue determinar la influencia del clima afectivo en el trabajo cooperativo que realizan los docentes en el quehacer académico y administrativo. El estudio se desarrolló con el enfoque cuantitativo y con un diseño no experimental de corte transversal y correlacional. La muestra conformada por 6o docentes entre niveles de primaria y secundaria. Se utilizó instrumentos estandarizados y para los resultados inferenciales se utilizó la prueba de Pearson con la que se demuestra que hay influencia entre variables, pero no es significativa. Se concluye que el trabajo cooperativo puede estar influenciado por otros factores no necesariamente por el clima afectivo.
\end{abstract}

Palabras clave: Clima afectivo, trabajo cooperativo, docentes, instituciones educativas. 


\begin{abstract}
This affective climate research, focused on the cooperative work of teachers, resulted from the concerns of educational institutions with respect to the responsibilities of their educational agents. The institutions were interested in determining the influence of the affective climate on the cooperative work of teachers as they completed their academic and administrative tasks. This study applied a quantitative approach and a non-experimental cross-sectional and correlational research design. The sample consisted of 60 teachers across primary and secondary levels. Standardized instruments were used and for the inferential results the Pearson test was used; when there was influence between variables, although the correlations were not significant. Cooperative work may be influenced by factors other than the affective climate.
\end{abstract}

Key words: Affective climate, cooperative work, teachers, educational institutions.

\section{Introducción}

Uno de los resultados más sobresalientes del cambio ha sido fomentar en los equipos de trabajo una estructura primordial de realización y gestión laboral (Peiró y Munduate, 1999). Las bases tradicionales, con respecto al concepto de puesto, son cambiadas por estructuras descentralizadas, horizontales y en forma de red, cuyos nódulos gozan de un grado elevado de autonomía y se encuentran interconectados; ya que un 80\% de los trabajadores son autónomos por la confianza y el clima que se practica en la institución y ello genera un trabajo de equipo en cada uno de sus miembros, que colabora en pro de los objetivos institucionales. En el actual contexto organizacional, los equipos de trabajo obtienen un estatus diferenciado en la competencia organizacional y se establecen como unidad básica de la institución.

Por otro lado, la teoría e investigación multinivel indaga acerca de las consecuencias del afecto en las organizaciones, postulando que este se manifiesta en cinco niveles diferenciados, a nivel intrapersonal, interpersonal, interaccional, grupal y organizacional (Ashkanasy, 2002). Agregado a ello, se destaca la importancia de las experiencias afectivas de los integrantes del equipo, que acoplados, desencadenan un estado afectivo masivo que influiría en cada uno de sus miembros (De Dreu, West, Fischer y MacCurtain, 2001). Así, cada uno de sus miembros colabora y coopera con entusiasmo y agrado a la institución. Par el caso de las instituciones educativas, los educadores y los directivos se relacionan en torno al factor organizacional. Este tipo de conexiones se encuentran estructuradas y unidas a alguna clase de regulación, que es identificada y admitida por ambos, con el objetivo de desarrollar el aprendizaje dentro de un clima socio emocional efectivo.

A partir de los años ochenta, se ha platicado mucho acerca de enseñanza 
estratégica, modelamiento, tutoreo, etc. Además, en paralelo a lo cognitivo, en la última década, se ha incrementado la tendencia de los profesores acerca del impacto que poseen las dimensiones afectivas en el desempeño docente. Éstas se clasifican en: 1) habilidad para percibir emociones y sentimientos en uno y en terceros, y 2) habilidad para emitirlos de modo apropiado y autorregulado en la interacción con los educandos. Las anteriores habilidades son las que Howard Gardner conoce como inteligencia intra e interpersonal y que subsiguientemente fueron denominadas por Goleman (1995) como inteligencia emocional, la cual es considerada esencial para propagar climas sociales propicios para el aprendizaje.

Determinar los indicadores de ocurrencia del clima afectivo en la institución educativa, en la cual los directivos deben ser los conductores hacia los docentes y trabajadores de la institución que conduzca a responsabilidad, respeto y armonía; creando un clima afectivo conformado por diversos factores, que interactúan entre sí, principalmente relacionados con factores afectivos y emocionales; de modo que el director o administrador es el encargado de patrocinar un atractivo clima afectivo, caracterizado por ser ameno, libre, sin perjuicios y tolerante. Al lograr ello en las instituciones, estamos seguros que los trabajadores, en esta oportunidad docentes, realizarán su trabajo con empeño, haciendo que se involucren en los trabajos en equipo.

Esta investigación tuvo como propósito establecer si existe relación entre el clima afectivo y el trabajo cooperativo de los docentes de una institución educativa, en la que se identificó que la comunicación que se recibe y reproduce entre los agentes educativos no es la correcta. Asimismo la integración es muy pobre, sobre todo si nos referimos a la totalidad de sus integrantes, donde los docentes demuestran inseguridad en sus acciones; no se encuentran predispuestos a realizar actividades, lo que puede tener relación con la conducción o dirección de la institución, ya que está dirigida por personas que no se preocupan por la institución. La mayor parte de las decisiones son pensadas en intereses personales más no institucionales, encontrando desacuerdos entre sus miembros, y esto genera desconcierto en el personal, ya que con dichas acciones crea un ambiente de desconfianza. A ello sumamos la falta compromiso del director, quien se ausenta de la institución, delega funciones a los coordinadores y no muestra interés en el trabajo en equipo.

\section{Empleando un enfoque colectivo al estudio del afecto}

Dentro de análisis psicosocial en el espacio laboralista y organizacional, se ha focalizado el estudio de los fenómenos a nivel individual, donde se considera que un individuo recopila y procesa datos formando experiencias afectivas personales sin correspondencia con los estados afectivos del grupo; proporcionando información acerca del funcionamiento de los individuos, las organizaciones y el trabajo. A pesar de ello, no explica dequé manera se llevan a cabo los afectosy otras experiencias de equipo de las 
áreas de trabajo. Relacionar a las personas, las organizaciones y el trabajo es complejo, y más aún si le sumamos los afectos individualesy colectivos en el desempeño laboral, y su relación con el trabajo cooperativo, que es materia de investigación en la actualidad porque permite ver el lado humano en el desarrollo de una organización.

Actualmente, los estudios psicosociales y organizacionales están interesados por comprender y valuar fenómenos de equipo de trabajo (González-Romá, Peiróy Tordera, 2002), las manifestaciones personales que se dan lugar en el área de trabajo y que, a su vez, dan origen a fenómenos a nivel colectivo. Si bien es cierto que la conducta humana se estructura socialmente, ésta también se caracteriza por la regularización social. Incluso la conducta, las percepciones y las experiencias de sujetos aislados están influidas por conceptos, valores e ideas que emergen del medio social y que se comparten, con otros individuos (Kozlowski y Klein, 200o). Así, la interacción humana dentro de una organización laboral comprende comportamientos, percepciones y vivencias individuales y colectivas que surgen del entorno en el cual se desenvuelve. En las investigaciones que abarcan el afecto dentro de lugares de trabajo, se tiende a considerarlo como una pertenencia colectiva de las áreas de labor. La investigación que se lleva cabo, enfoca desde una aproximación colectiva, el análisis del afecto de los colaboradores que laboran en equipos de trabajo dentro de la organización, aspectos que son importantes debido a su utilidad para entender la vida y el desarrollo del trabajo en las empresas.

\section{Modelo multinivel de las experiencias afectivas en la organización}

El modelo multinivel del estudio del afecto toma en cuenta todas las manifestaciones posibles de las personas en relación con la organización y funciones que desempeña. Ashkanasy (2003) refiere que este modelo está conformado por niveles: el nivel intrapersonal incorpora los procesos neurofisiológicos, conteniendo las manifestaciones fisiológicas de las emociones y los estados de ánimo. Estos estados afectivos son abordados desde la teoría de los sucesos afectivos (Weiss y Cropanzano, 1996), la cual considera que las experiencias afectivas en el centro laboral están definidas, tanto por las disposiciones afectivas personales y los sucesos laborales discretos que percibimos por experiencia en nuestra actividad laboral. Cada uno de estos sucesos ocasiona reacciones afectivas determinadas, las cuales intervienen en el comportamiento de los colaboradores. La diversidad de estos estados traerá como efectos, múltiples resultados en el centro laboral. Según esta teoría, nuestras conductas y actitudes se ven afectadas por las experiencias y vivencias que experimentamos dentro del lugar de trabajo.

En el nivel interpersonal, se tiene en cuenta las diferencias personales, y se sitúa aquí los rasgos afectivos, la satisfacción, el burnout, el compromiso afectivo y la inteligencia emocional. Dichos factores de carácter afectivo provienen de las manifestaciones intrapersonales y se mostrarían como efectos particulares. En este nivel, es importante tener claro el nivel intrapersonal, y conocer bien 
nuestras emociones y reacciones, para poder interrelacionarse con los demás.

El nivel interaccional, considera a la percepción y comunicación de las experiencias afectivas de dos personas. Waldrom (2000) remarca que este nivel es el núcleo afectivo en los centros de trabajo, debido a su importancia como enlace entre los niveles uno y dos, por una parte, y los niveles cuatro y cinco, por otra. Este nivel es el que permite conectar con los demás niveles incluyendo la percepción y comunicación de las experiencias de tipo afectivo.

El nivel de equipo, identifica a los grupos laborales como una "incubadora afectiva”. En donde los equipos experimentan afectos combinables entre sí y generan un estado afectivo colectivo que actuará en cada uno de los integrantes. Kelly y Barsade (2001) mencionan que los grupos de trabajo tienen una específica composición afectiva (o estado de ánimo), que influencia en las particularidades afectivas de los integrantes. Desde ese momento, este tipo de afecto se lleva a cabo por medio de diversos procesos grupales. En este nivel nace el intercambio afectivo de equipos, dando lugar al concepto de inteligencia emocional grupal que se forma en base a las características afectivas del equipo con los aportes de cada miembro.

El nivel organizacional, es de carácter cualitativo, pues en los niveles anteriores los valores y políticas de la organización eran analizados por los colaboradores en relaciones directas con sus pares y sus superiores. A este nivel, el escenario es poco claro y las interacciones de los colaboradores con los demás integrantes de la organización son cortas y menos frecuentes, pues aquí se manejan conceptos de "clima afectivo" o el de "cultura afectiva". El primero de ellos hace referencia al estado de ánimo colectivo relacionado al entorno laboral, la organización y su administración; siendo considerado un fenómeno afectivo. De otro lado la cultura está enfocada en las creencias y valores. Este nivel organizacional no solo involucra el estado de ánimo en relación del entorno laboral sino también considera las creencias y valores que poseen los miembros de la organización.

Cooper y Williams (1994) manejan el concepto de "organización saludable", dentro de este nivel, la cual es definida como la organización que prioriza la comodidad de los colaboradores y la conservación de efectos positivos en la organización. Cuando hacemos referencia al clima organizacional debemos tener en cuenta que existen dentro de las organizaciones sub climas (climas diferentes), y utilizamos el concepto de clima afectivo por asociarlo con el trabajo, la organización y la gestión dentro de un ambiente laboral.

Para la presente investigación nos ubicaremos en el cuarto nivel del modelo de Ashkanasy (2003) y emplearemos el equipo de trabajo como foco de estudio de los climas afectivos. 


\section{Definiciones de clima afectivo}

De Rivera (1992) lo conceptualiza como un fenómeno grupal que puede ser percibido he interpretado de modo objetivo. Este concepto podría utilizarse de modo ventajoso para investigar el afecto en los equipos y en las organizaciones, nos permite entender cómo las emociones pueden intervenir en nuestro desenvolvimiento, dentro de un grupo social.

Para Franco (1998), el clima afectivo implica "instaurar fiscalizadores de orden emocional que nos permitan revelar formas de aceptación social para expresar sus emociones" (p. 141). Pues al conocer el carácter de los individuos, ello nos lleva a conocer sus emociones, y por ende a mejorar su modo de actuar frente a su entorno; permite además, controlar las emociones, para así descubrir las formas de expresarlas de manera aceptable y que a su vez ayuda a desarrollar y mejorar la personalidad.

Para el estudio las experiencias afectivas colectivas serán conocidas como "clima afectivo". Este concepto es un constructo perteneciente al área organizacional y se utiliza para referirse a las percepciones que comparten los colaboradores de una organización. Por ende, el "clima emocional" y el "clima afectivo" cobran fuerza e la actualidad, pues presentan gran importancia dentro del ambiente organizacional (De Rivera, 1992).

Pirola-Merlo et al. (2002) destacan que el tono afectivo y clima afectivo, son cotejables debido a que explican las experiencias afectivas que poseen en común los integrantes de una unidad. González-Romá, Peiró, Tordera y Belmonte (200o) hacen referencia a "afecto" como un concepto más amplio que incluye nociones afectivas: estados de ánimo, emociones, sentimientos, disposición afectiva, etc., conceptualizándolo como un estado de sentimiento subjetivo que comprende estados de ánimo poco claros hasta emociones intensas (Ashforth y Humphrey, 1995). Así, utilizar el término afecto dentro de una organización incluye conocer estados de ánimo, emociones, sentimientos, etc., de las personas que forman parte de la misma. El concepto de clima afectivo se refiere a "los estados de ánimo compartidos por integrantes de un equipo de trabajo" (Gamero, 2008, p. 58), de modo que el clima afectivo permite que los miembros de un equipo de trabajo aprendan a compartir sus estados de ánimo y emociones.

\section{Componentes del clima afectivo}

Según Gamero (2008) tenemos dos dimensiones del clima afectivo: la intensidad y la homogeneidad.

\section{Intensidad del clima afectivo}

Comprendida como los estándares de los estados de ánimo de los miembros de un equipo, que miden la fuerza o energía con que se realiza una actividad, un sentimiento o una emoción. Asimismo, la intensidad del clima afectivo se puede ver afectada por dos tipos de conflicto, el relacional y el conflicto de tarea. 


\section{Conflicto relacional}

Gamero (2008) cita a Jehn (1994) al referirse a esta dimensión, que inserta elementos personales y afectivos como fricción, tensión y aversión que se da en los integrantes del equipo. Así pues, dentro de un conflicto relacional intervienen aspectos personales y afectivos que componen el grupo. Cuando en el entorno se observa un conflicto relacional, la información acerca de políticas, prácticas y procedimientos que vienen a ser premiadas, respaldadas y esperadas por la empresa, afectarán el desarrollo del clima afectivo (De Dreu y van Viannen, 2001).

\section{Conflicto de tarea}

Para Gamero (2008) quien cita a Jehn (1994), este conflicto implica discrepancias en ciertos aspectos y opiniones referentes a la tarea y funciones. Este se manifiesta dentro de un grupo o equipo cuando no existe acuerdos entre los colaboradores con respecto a las labores realizadas. Hay que considerar que ante el desarrollo de una tarea siempre se presentan diferencias, porque no todos percibimos las cosas de igual forma, aunque lo ideal es coordinar para una buena realización de la tarea destinada.

\section{Homogeneidad del clima afectivo}

Gamero (2008) la define como el grado de convergencia o semejanza de los estados deánimo de los miembros de un equipo, y dependen de los siguientes aspectos:

\section{Interacción entre los miembros del equipo}

Es la relación recíproca por medio de la cual gran parte de los integrantes articulan su comportamiento con respecto a la la transmisión de mensajes o informaciones. Estos podrían llevarse a cabo a través de medios personalizados. Los miembros de un equipo deben interactuar acoplando sus conductas de acuerdo al entorno y transmitir mensajes e informaciones entre ellos.

Normas de regulación afectiva

Se refiere a la graduación del comportamiento. Este ajuste de control y modulación de respuestas afectivas se ha investigado con mayor impacto en la primera infancia (Goleman, 1995); sin embargo, la regulación afectiva se manifiesta en el transcurso del desarrollo humano, debido a que concierne con el adecuado contacto con uno mismo, con el entorno y con la capacidad de simbolización. Regular lo afectivo involucra saber controlar el comportamiento y las respuestas afectivas, aprendizajes que se dan a lo largo de nuestro desarrollo personal y la relación con nuestro entorno.

\section{Comparación afectiva}

Abarca las reacciones emocionales personales en el entorno laboral comparándola con las reacciones emocionales de otros integrantes del equipo, y mediante este proceso se forma parte del equipo al cual pertenecemos. 


\section{Rendimiento del equipo}

En el último decenio, los estudios ejecutados en el área de la psicología organizacional se vienen desarrollando desde un aspecto "micro" para analizar el comportamiento del individuo (Peiró, 2009). A pesar de ello, la dinámica organizacional es compleja y por ende se han desarrollado enfoques, teorías, modelos, conceptos e indagaciones que abarcan niveles de análisis diferenciados.

Gamero (2008) se refiere al rendimiento del equipo como la autogestión de los equipos de trabajo para desarrollar estrategias innovadoras y alcanzar resultados diferenciados, y con ello, estándares elevados de rendimiento que los que se consigue por medio de los procedimientos tradicionales. En ese sentido, la convicción del equipo es muy importante para obtener niveles elevados en el rendimiento del mismo.

\section{Fundamentos teóricos del trabajo cooperativo}

El trabajo cooperativo ha posibilitado el intercambio de interdependencias y conocimientos, la socialización de procesos, experiencias y los resultados adquiridos; que traen como consecuencia el desarrollo integral del ser humano, siendo lo que le ha permitido avanzar el ser un ser social por naturaleza, modificando su entorno de manera social e individual. En ese sentido, todo conocimiento adquirido por el ser humano es elaborado socialmente a través de la interacción entre individuos con su medio, dependiendo de su contexto histórico;y transmitido de una generación a otra a través de la incorporación de las futuras generaciones dentro de la sociedad, siendo la educación fundamental para esto. Los antecedentes de aprendizaje cooperativo forman parte de la historia de la pedagogía y la humanidad. Así pues, tanto Piaget (1981) como Vigotsky (1987) dicen que los individuos cooperan cuando surge un conflicto socio-cognitivo que genera un desequilibrio cognitivo que, al mismo tiempo, estimula el desarrollo del conocimiento y la habilidad de adoptar diferentes puntos de vista.

\section{Trabajo cooperativo}

Según Ovejero (1999) es "una técnica educativa que busca la mejora del rendimiento y potenciar las capacidades intelectuales y sociales de los educandos" (p. 46). Es también una estrategia de gestión que beneficia la organización de los alumnos en grupos heterogéneos para llevar a cabo funciones y actividades de aprendizaje, y que involucra agrupar a los estudiantes en pequeños equipos con el objetivo de potenciar el desarrollo de sus miembros. Así, el trabajo cooperativo permite extraer el potencial de cada miembro y fortalecer el objetivo del trabajo a realizar.

Es por ello, que Ferreiro y Calderón (2001), señalan que el aprendizaje cooperativo:

Acrecienta la relación entre los miembros del equipo, con el docente y los otros equipos, de modo que cada uno aprende el contenido asignado y a su vez, el grupo también, formulando una 
manera diferente de relacionarse maestro y alumno en el proceso de enseñar y aprender. (p. 31)

El trabajo cooperativo es además, un valor incorporado en el currículo de formación, necesario para la mediación educativa en el aula y como ingrediente esencial de la organización de la comunidad educativa (Klein, Kuh, Chun, Hamilton y Shavelson, 2015). Por ello, el trabajo cooperativo es un valor importante en la labor educativa que requiere compromiso de los integrantes de la comunidad educativa.

El trabajo cooperativo demanda de una planificación minuciosa y exhaustiva de la intervención para lograr las condiciones competentes que necesita esta metodología (Cabrera, Colbeck y Terenzini, 2001). Aplicar el trabajo cooperativo necesita de una preparación que permita lograr los objetivos planteados. Este tipo de aprendizaje supone la "utilización didáctica de equipos reducidos en los que los estudiantes trabajan para potencializar su propio aprendizaje y el de los otros" (Johnson, Johnson y Holubec, 1999); y así, este método busca optimizar el aprendizaje de cada miembro que compone el grupo.

Pújolas (2008) subraya que los integrantes del equipo poseen una doble responsabilidad: aprender la enseñanza del docente y contribuir a que lo aprendan su equipo. Los docentes usan este tipo de aprendizaje para lograr que los estudiantes aprendan los contenidos de la disciplina y para que aprendan a trabajar en equipo como competencia profesional. Es decir "cooperar para aprender y aprender a cooperar". Además, el aprendizaje cooperativo no solo busca aprender los conocimientos que se reciben, sino también compartir lo que se aprenda con los otros miembros del equipo. Así, la cooperación es compartir actividades entre los integrantes para la consecución de una meta en común. El aprendizaje personal se logra si todos y cada uno de los integrantes lo han logrado también. Cooperar es interactuar entre los miembros del equipo para alcanzar el logro de la meta trazada.

Para Rué (1994) es “una herramienta de enseñanza que tiene su origen en la distribución de la clase en pequeños equipos mixtos en la cual se trabaja conjunta y coordinadamente entre sí para dar solución a actividades académicas y profundizar en su propio aprendizaje" (p. 120). Para Jonhson et al. (1999) son métodos de instrucción para grupos pequeños, de entrenamiento y desarrollo de habilidades diferenciadas (aprendizaje y desarrollo personal social), en la cual cada integrante es responsable de su aprendizaje y de los miembros de su equipo. Así, existe una responsabilidad entre el aprendizaje personal y el aprendizaje grupal que permite el desarrollo de habilidades mixtas. Por ello, este tipo de trabajo se elabora teniendo en cuenta la interacción de los miembros y también el trabajo individual que cada uno realiza.

Según Giraldo (2007), el aprendizaje cooperativo tiene su fundamento básico en la valoración del potencial educativo y la interacción grupal, y tiene en cuenta los valores de socialización e integración 
para el aumento del rendimiento escolar. Este autor nos hace percibir que el aprendizaje cooperativo gira entorno a las relaciones interpersonales de los miembros del grupo, las cuales si son eficaces permiten que se obtenga buenos resultados. En un trabajo cooperativo hay diversos grados de coordinación, planificación e interacción entre los individuos que cooperan, por ello se deben tomar en cuenta estos aspectos.

\section{Generalidades sobre el trabajo de grupo}

García, González y Mérida (2012) indican que este tipo de actividad es importante para el desarrollo cognitivo, social y académico; y permite potenciar las relaciones humanas y el aprendizaje autónomo. Así, el trabajo en grupo nos ayuda al desarrollo cognitivo, social y académico, así como también a potenciar las interacciones sociales

\section{Organización del trabajo en grupo}

Para García, González y Mérida (2012) es la planificación de los detalles en los grupos por parte del educador. Esta nos permite identificar el punto de vista que poseen los estudiantes en referencia a lo ejecutado por el docente. Objetivamente se analiza si la cantidad, dificultad, planificación y seguimiento de todo lo propuesto tiene concordancia con la consecución de aprendizajes. En consecuencia, la formación del trabajo cooperativo debe estar sustentado en una buena planificación, que pueda tomar en consideración la opinión de los participantes, y debe contar además, con normas claras.

\section{Funcionamiento de los grupos de trabajo}

Aquí se incorporan cuestiones que tienen relación con una serie de acciones ejecutadas en el transcurso del trabajo grupal. Se pone interés por las actividades que realiza el alumno antes de la preparación del resultado, y se agregan cuestiones que hacen referencia a la ponderación del trabajo en grupo con respecto a la evaluación final, información de criterios utilizados, la diferenciación en la valoración de las distintas aportaciones personales, autoevaluación de los alumnos y la valoración entre iguales. Así, en el funcionamiento de los grupos de trabajo no solo se ve el desarrollo, la ejecución y los resultados sino también la evaluación general y autoevaluación y la evaluación entre pares.

\section{Metodología}

Esta investigación se desarrolló según una metodología cuantitativa y se aplicó un diseño no experimental, de corte transversal correlacional, porque el estudio está centrado en identificar la relación del clima afectivo y el trabajo cooperativo en instituciones educativas.

\section{Muestra}

La muestra de estudio estuvo conformada por 6o docentes de una institución educativa de distrito del Rímac, tanto de nivel primario como secundario. Para la delimitación de los docentes integrantes de la muestra se utilizó el criterio de inclusión constituido por 
docentes nombrados con 10 años de experiencia mínima en instituciones educativas, porque son ellos quienes se rehúsan a trabajar en equipo.

\section{Instrumentos}

Para el recojo de información se utilizaron dos instrumentos: la Escala del Clima afectivo de Gamero (2008), que cuenta con 40 ítems distribuidos en dos dimensiones. La primera denominada intensidad del clima afectivo y la segunda homogeneidad del clima afectivo, con índices y rango variados que a continuación detallamos: de la pregunta 1 al 12 las escalas son: Nada (1), Poco (2), Medio (3), Bastante (4) y Mucho (5). Asimismo, de la 13 al 30 las escalas son: Nunca (1), Pocas veces (2), Frecuencia media (3), Bastantes veces (4) y Muchas veces (5); mientras que del ítem 31 al 38 los rangos de respuesta son: Totalmente en desacuerdo (1), Bastante en desacuerdo (2), Algo en desacuerdo (3), Algo de acuerdo (4), Bastante de acuerdo (5) y Totalmente de acuerdo (6). Por último, del ítem 39 al 40 sus escalas son: Muy mal (1), Mal (2), Regular (3), Bien (4) y Muy bien (5).

El trabajo cooperativo fue evaluado por medio del Cuestionario ACOES de García, González y Mérida (2012), que está conformado por tres dimensiones: la primera nombrada Generalidades sobre el trabajo en grupo, la segunda referida a la Organización del trabajo en grupo, y la tercera es el Funcionamiento de los grupos de trabajo. Tiene a una escala ordinal de respuesta, cuyos valores para la interpretación son: $1=$ Totalmente en desacuerdo, 2= En desacuerdo, $3=$ Medianamente de acuerdo, 4= De acuerdo y $5=$ Totalmente de acuerdo.

Los instrumentos han demostrado validez y confiabilidad en varios estudios como el de Aguilar y De la Cruz (2017), Medina (2016) y Rengifo (2015) para el clima afectivo, así como de Díaz (2014) y Nuria (2007) para el trabajo cooperativo. En esta investigación la validez de contenido fue realizadas a través del juicio de expertos y la fiabilidad del clima afectivo fue de .741 y la del trabajo cooperativo de .926 .

\section{Procedimientos}

En el proceso para recolectar la información se diálogo con las autoridades de la institución educativa para solicitar la autorización pertinente y poder realizar la recolección. Para el análisis de los datos de utilizó el paquete estadístico SPSS versión 24. Para la prueba de la normalidad se empleó el estadístico Kolmogorov-Smirnov y para la contrastación de hipótesis se utilizó la correlación de Pearson.

\section{Resultados}

Posteriormente del trabajo de campo llegamos a resultados descriptivos e inferenciales de las variables estudiadas que a continuación detallamos. 
Tabla 1. Clima afectivo y trabajo cooperativo en docentes de las instituciones educativas del Rímac

\begin{tabular}{|c|c|c|c|c|c|c|c|c|}
\hline \multirow{3}{*}{ Clima afectivo } & \multicolumn{6}{|c|}{ Trabajo cooperativo } & \multirow{2}{*}{\multicolumn{2}{|c|}{ Total }} \\
\hline & \multicolumn{2}{|c|}{ Bajo } & \multicolumn{2}{|c|}{ Moderado } & \multicolumn{2}{|c|}{ Alto } & & \\
\hline & $\mathbf{n}$ & $\%$ & $\mathbf{n}$ & $\%$ & $\mathbf{n}$ & $\%$ & $\mathbf{n}$ & $\%$ \\
\hline Bajo & $\mathrm{O}$ & $0 \%$ & o & o\% & 6 & $10 \%$ & 6 & $10 \%$ \\
\hline Moderado & o & o\% & 15 & $25 \%$ & 39 & $65 \%$ & 54 & $90 \%$ \\
\hline Alto & o & o\% & o & o\% & o & o\% & o & о\% \\
\hline Total & $\mathbf{o}$ & о\% & 15 & $25 \%$ & 45 & $75 \%$ & 60 & $100 \%$ \\
\hline
\end{tabular}

En la tabla 1, se observa que el $65 \%$ de los docentes presentan un nivel moderado de clima efectivo y a la vez presentan un nivel alto de trabajo cooperativo, por lo que podemos afirmar que mientras haya clima afectivo mejor se presenta el trabajo cooperativo.

Tabla 2. Coeficiente de correlación entre clima afectivo y el trabajo cooperativo de los docentes de las instituciones educativas del Rímac

\begin{tabular}{|cccc|}
\hline & Clima afectivo & $\begin{array}{c}\text { Trabajo } \\
\text { cooperativo }\end{array}$ \\
\hline $\begin{array}{c}\text { Clima } \\
\text { afectivo }\end{array}$ & Correlación de Pearson & 1 & .079 \\
& Sig. (bilateral) & & .550 \\
Trabajo & Correlación de Pearson & .079 & 60 \\
cooperativo & Sig. (bilateral) & .550 & 1 \\
& $\mathrm{~N}$ & 60 & 60 \\
\hline
\end{tabular}

Los resultados de la Prueba de correlación de Pearson, arrojaron un coeficiente de correlación de $\mathrm{r}=.079$, lo que indica una correlación positiva despreciable. Además, el valor de significación observada $\mathrm{p}=.550$ es mayor al valor de significación teórica $\mathrm{p}=.05$. En consecuencia, la relación no es significativa al $95 \%$ y no se rechaza la hipótesis nula (Ho) asumiendo que existe relación directa pero no significativa entre el clima afectivo y el trabajo cooperativo de los docentes de las instituciones educativas del Rímac.

\section{Discusión}

Los resultados de nuestra investigación muestran que la relación entre el clima afectivo y el trabajo cooperativo de los profesores evaluados no es significativa, por lo tanto, se rechaza la hipótesis de investigación; en contraposición a lo mencionado por Díaz (2014) quien 
concluye que existe relación entre ambas variables. Rengifo (2015) por su parte, también concluyó que el clima afectivo de profesores universitarios de Iquitos, está relacionado significativamente con la motivación al logro profesional.

Los resultados de la investigación también muestran que el 90\% de los docentes tienen un nivel moderado de clima afectivo, y en concordancia a lo referido Medina (2016), se considera que para desarrollar el clima afectivo es necesario instaurar controles emocionales en las personas que les permitan descubrir formas aceptables de regulación que les ayuden a expresar sus emociones e interactuar con otros individuos.

De Rivera (1992) refiere que las experiencias afectivas son importantes en una institución, y siempre son un elemento fundamental en el área organizacional. Es por ello que, en esta investigación, las percepciones de unos u otros docentes, varían respecto a la intensidad del clima afectivo. Si bien en general hay un clima afectivo aceptable debido a la realización de los trabajos en común, el $40 \%$ de los docentes no percibe el clima afectivo como una prioridad para el cumplimiento de sus funciones.

Asimismo, en lo que respecta a los resultados descriptivos el $75 \%$ de los docentes perciben que en la institución educativa presenta un alto nivel de trabajo cooperativo, y en concordancia a ello Ovejero (1999), se concluye que la imagen de la escuela o institución educativa se ve reflejada según el contexto social. En consecuencia, en la institución educativa se presentan dificultades para conseguir una satisfactoria cohesión grupal, con las implicaciones que ello tiene tanto para el trabajo escolar. Si tenemos en cuenta que el trabajo cooperativo es fundamental para el logro de los objetivos institucionales, éste podría ser un valor añadido al currículo formativo, y un elemento imprescindible tanto para la intervención educativa en un aula como para la labor docente como miembro de una comunidad educativa con la que debe estar comprometido (Klein, Kuh, Chun, Hamilton y Shavelson, 2015).

En el estudio se observa finalmente, que el $87 \%$ de los docentes presentan un nivel alto de concepción del trabajo en grupo puesto que los docentes consideran que es necesario planificar y organizarse para la distribución equitativa de las funciones; y asimismo, mantener una comunicación clara y oportuna para el éxito de las actividades. En respaldo a ello, Ovejero (1999), hace mención que el trabajo cooperativo demanda de una planificación minuciosa y rigurosa de la intervención para lograr las condiciones idóneas que precisa esta metodología, condiciones que en la institución educativa, no siempre tienen lugar ya que se conforman los grupos no por afinidad más bien por necesidad; y sin tomar en cuenta aspectos de perfil idóneo en múltiples responsabilidades. A pesar de ello, el 58\% de los docentes perciben que la organización del trabajo en grupo es aceptable. 


\section{Referencias}

Aguilar. R., \& De la cruz, J. (2017). Clima afectivo en los docentes de la Facultad de Trabajo Social de la Universidad Nacional del Centro del Perú. (Tesis profesional). Universidad Nacional del Centro del Perú, Huancayo, Perú.

Ashforth, B., \& Humphrey, R. (1995). Emotion in the workplace: A reappraisal. Human Relations, 48, 97-125.

Ashkanasy, N. (2002). Studies of cognition and emotion in organizations: Attribution, affective events, emotional intelligence and perception of emotion. Australian Journal of Management, 27, 11-20.

Ashkanasy, N. (2003). Emotions in organizations: A multi-level perspective. En F. Dansereau y F. U. Yammarino (Eds.), Research in Multi-level Issues, Vol. 2: Multilevel Issues in Organizational Behavior and Strategy (pp. 9-54). Amsterdam: Elsevier Science.

Cabrera, A., Colbeck, C., \& Terenzini, P. V. (2001). Developing performance indicators for assesing classroom teaching practices and student learning. Research in Higher Education, 42(3), 327-352.

Cooper, C. L., \& Williams, S. (1994). Creating Healthy Work Organizations. Chichester, UK: Wiley.

De Dreu, C. K. W., \& Van Vianen, A. E. M. (2001). Managing relationship conflict and the effectiveness of organizational teams. Journal of Organizational Behavior, 22, 309-328.

De Rivera, J. (1992). Emotional climate: Social structures and emotional dynamics. International Review of Studies on Emotions, 2, 197-218.

Díaz, S. (2014). Comunicación Organizacional y Trabajo Cooperativo en Trabajadores de una Universidad de la Ciudad de Quetzaltenango. (Tesis profesional). Universidad Rafael Landívar, Quetzaltenango, Guatemala.

Ferreiro, G. R., \& Calderón, E. M. (20o1). El ABC del Aprendizaje Cooperativo. Trabajo en equipo para enseñar y aprender. México. Editorial Trillas.

Franco, T. (1998). Vida afectiva y educación infantil. España: Narcea, S.A. 
Gamero, N. (2008). El clima afectivo en equipos de trabajo: antecedentes y consecuencias. España: Universidad de Valencia.

García, M., González, I., \& Mérida, R. (2012). Validación del cuestionario de evaluación ACOES. Análisis del trabajo cooperativo en educación superior. Revista de Investigación Educativa, 3o(1), 87-109.

Giraldo, C. (2007). ¿Protección o desprotección social? Bogotá: Universidad Nacional de Colombia.

Goleman, D. (1995). Inteligencia Emocional. Barcelona: Kairós.

González-Romá, V., Peiró, J. M., Tordera, N., \& Belmonte, J. (200o). Emotional climates at work. Determinants of the formation processes of collective emotions. Trabajo presentado en el XXVII International Congress of Psychology, Estocolmo, Suecia.

González-Romá, V., Peiró, J. M., \& Tordera, N. (2002). An examination of the antecedents and moderator influences of climate strength. Journal of Applied Psychology, 87, 465-473.

Johnson, D. W., Johnson, R. T., \& Holubec, E. J. (1999). El aprendizaje cooperativo en el aula. Buenos Aires: Paidós.

Kelly, J.R., y Barsade, S.G. (2001). Mood and emotion in small groups and workteams. Organizational Behavior and Human Decision Processes, 86, 99-130.

Klein, S. P., Kuh, G., Chun, M., Hamilton, L., \& Shavelson, R. (2005). An Approach to Measuring Cognitive Outcomes across Higher Education Institutions. Higher Education, 46(3), 251-276.

Kozlowski, S. W., \& Klein, K. J. (200o). A multilevel approach to theory and research in organizations: Contextual, temporal, and emergent processes. En K.J. Klein y S.W. Kozlowski (Eds.), A Multilevel Theory, Research, and Methods in Organizations. Foundations, Extensions and New Directions (pp. 3-9o). San Francisco: Jossey-Bass.

Medina, C. (2016). Relación entre el Clima Afectivo y la Satisfacción Laboral de Profesores de un Centro Educativo Adventista de la Región de Junín (Tesis profesional). Universidad Peruana Unión, Junín, Perú.

Nuria, V. (2007). Clima afectivo y equipos de trabajo: antecedentes y consecuencias (Tesis doctoral). Universidad de Valencia, Valencia, España. 
Ovejero, A. (1999). El Aprendizaje Cooperativo: Una alternativa eficaz a la enseñanza tradicional. Barcelona: PPU.

Peiró, J. M., \& Munduate, L. (2001). Research developments on Workand Organizational Psychology in Spain: An overview. Revista de Psicología Social Aplicada, 11, 31-48.

Peiró, S. (2009). Valores educativos y convivencia. Ecuador: Club Universitario.

Piaget, J. (1981). La teoría de Piaget. Infancia y Aprendizaje, 4(2), 13-54.

Pirola-Merlo, A., Härtel, C., Mann, L., \& Hirst, G. (2002). How leaders influence the impact of affective events on team climate and performance in $\mathrm{R} \& \mathrm{D}$ teams. The Leadership Quarterly, 13, 561-581.

Pújolas, P. (2008). Nueve ideas clave del aprendizaje cooperativo. Barcelona: Grao.

Rengifo, A. (2015). Clima afectivo y la motivación al logro profesional docente en la Facultad de Ciencias Biológicas. (Tesis de Maestría). Universidad Nacional de la Amazonía Peruana, Iquitos, Perú.

Rue, J. (2010). El trabajo Cooperativo. (Documento en formato html). Descargado el 5 de julio de 2018 de: .Giac.upc.es/pag/giac_cas/giac_que_es.htm.

Vigotsky, L. (1987). El desarrollo de los procesos psicológicos superiores. México: Editorial Grijalbo.

Waldrom, V. R. (200o). Relational experiences and emotion at work. En S. Fineman (Ed.), Emotion in Organizations (pp. 64-82). London: Sage Publishing.

Weiss, H. M., \& Cropanzano, R. (1996). Affective events story: A theoretical discussion of the structure, causes and consequences of affective experiences at work. En B. M Staw, y L. L. Cummings (Eds.), Research in Organizational Behavior, vol. 18 (pp. 1-74). Greenwinch, CT: JSI Press.

Recibido: 22 de abril de 2019

Aceptado: 15 de octubre de 2019 